\title{
Discurso Inaugural II Congreso Chileno de Salud Pública
}

\author{
GIORGIO SOLIMANO. DIRECTOR ESCUELA DE SALUD PÚBLICA. FACULTAD DE MEDICINA.
} UNIVERSIDAD DE CHILE

Un miércoles de julio de 2007, varios de los que nos encontramos aquí, estuvimos en este salón inaugurando el Primer Congreso Chileno de Salud Pública, el primer encuentro científico en el país en este campo. Sin duda, ese año marcó un hito en la salud pública nacional: más de 400 personas se reunían para compartir las más recientes investigaciones y experiencias, en un evento de gran nivel.

En aquella ocasión, anunciamos que el próximo Congreso se realizaría dentro de tres años, y que -en el intertanto- se mantendrían las Jornadas Chilenas de Salud Pública, que están próximas a cumplir 30 años.

$\mathrm{Y}$ aquí estamos, cumpliendo esa promesa y con la firme intención de reencontrarnos en tres años más. Hoy no son 400 los inscritos, sino 600. En estos dos días no se presentarán 290 trabajos, sino 339; hay nuevos rostros y nuevos socios de la Escuela de Salud Pública en esta iniciativa; hay nuevos conocimientos, nuevas experiencias y nuevos desafíos que les invito, cordialmente, a enfrentar juntos.

La salud pública en nuestro país es depositaria de una valiosa historia, la que aporta a la necesidad de continuar trabajando por mejorar las condiciones de salud de la población, adaptándonos a una realidad social, cultural, económica y epidemiológicamente siempre cambiante. Y este quehacer sólo es concebible a través de la conjunción de esfuerzos entre todos quienes, desde las más diversas disciplinas y ámbitos de acción, tenemos mucho que aportar.

La realización de este Congreso es una muestra de ello. Aquí, se plasma el compromiso de distintos actores por contribuir eficazmente a la protección y mejoramiento de la salud de las personas, las comunidades y su entorno, especialmente aquellas más vulnerables.
El Ministerio de Salud, la Organización Panamericana de la Salud y la Comisión Nacional de Investigación Científica y Tecnológica acompañan a la Universidad de Chile como convocantes. Por su parte, las universidades de Tarapacá, Antofagasta, Valparaíso y La Frontera, la Universidad Mayor, la Universidad de Santiago y la Pontificia Universidad Católica de Chile, también sumaron su experiencia y prestigio como integrantes del Comité Científico, mediante la participación de destacados académicos, a quienes agrademos su valiosa contribución.

El año del Bicentenario encuentra a nuestro país en un sitial de privilegio en el concierto mundial en cuanto a indicadores de salud. No obstante, junto a los avances científicos y tecnológicos, a la globalización económica, mejores niveles de educación, cambios demográficos y mejores estándares de vida de la población, el siglo XXI ha desnudado las grandes desigualdades que esconden los promedios, planteando una serie de retos que cobran especial relevancia en el sector salud.

Convivimos con un nuevo perfil epidemiológico, enfermedades emergentes y reemergentes con múltiples vías de propagación, catástrofes naturales, deterioro medio ambiental, nuevas formas de organización del trabajo y crecientes inequidades en el acceso a los bienes y servicios. Todo ello configura un panorama complejo y desafiante, particularmente para los responsables de las políticas de salud.

El Segundo Congreso Chileno de Salud Pública quiere dar cuenta de esta nueva agenda, con la cual enfrentamos el cambio de época. Con ese fin, el evento busca generar un amplio espacio para la difusión del conocimiento científico, así como para el debate interdisci- 
plinario de los temas relevantes -actuales y futuros- que atañen a la salud pública a nivel nacional y global, con los cuales nuestra Escuela está decididamente comprometida.

Es por ello que, además de la presentación de investigaciones y experiencias innovadoras procedentes de todo Chile y también de países como México, Cuba, Venezuela, Brasil, Perú, Alemania y otros, tendremos el privilegio de contar con conferencistas de renombre internacional, quienes nos hablarán de "Los desafíos para la salud pública contemporánea en tiempos de cambio", de la "Reforma de salud en Estados Unidos: un paso importante pero no suficiente" y del "Desarrollo y perspectiva de la salud en Chile: los retos del Bicentenario".

Pero el Congreso persigue, por sobre todo, constituir una plataforma para el fortalecimiento de la investigación en salud pública. Una investigación que, pese a algunos progresos, a nuestro juicio todavía tiene mucho por avanzar.

Podemos especular sobre las causas de ello, las que pueden ir desde los insuficientes recursos que históricamente se ha asignado a la investigación científica en Chile, al limitado reconocimiento que la salud pública recibe como disciplina científica, tanto en las instancias gubernamentales como al interior de las propias universidades. Esto, sin lugar a dudas, constituye una realidad que debemos revertir.

En este contexto, he estimado oportuno compartir con ustedes algunos resultados del estudio realizado en nuestra Escuela, financiado por el Programa Domeyko, "El estado del arte de la investigación en salud pública en Chile en los últimos 20 años", que será presentado en detalle en una de las sesiones.

Sobre institucionalidad, el estudio muestra que las universidades lideran la productividad con un $87 \%$ de las investigaciones; el sector público está muy atrás, con un $6,9 \%$, y luego siguen la sociedad civil y otros, con porcentajes ínfimos.

De las instituciones académicas, las universidades tradicionales son responsables de la mayor parte de la producción en investigación $\mathrm{y}$, entre éstas, la Universidad de Chile destaca con el 47\%, seguida de Universidad Católica, la de Concepción, la Universidad Austral y la de La Frontera. Es preocupante constatar que sólo el 55\% de las investigaciones generó publicaciones.

El financiamiento proviene de Conicyt, de fondos académicos, de recursos del sector público y privado nacional y de fondos extranjeros e internacionales.

En resumen, la institucionalidad de la investigación en salud pública en Chile se caracteriza por presentar una estructura heterogénea y disímiles niveles de productividad, tanto según el tipo de institución como entre instituciones de un mismo sector.

Según los investigadores entrevistados, las principales dificultades se relacionan con problemas de financiamiento, ya sea por la escasez de recursos disponibles o por la orientación de los mismos hacia otros tipos de investigación.

Otras limitantes son los problemas para constituir equipos de investigación, la carencia de un sistema de registro y administración de la información e indicadores de salud accesible para los investigadores, problemas para constituir alianzas y vínculos entre distintos sectores y la falta de tiempo para investigar. A ello se suma una débil valoración social de la salud pública y cierta incomprensión de la importancia de la investigación y de la salud pública, por parte de los tomadores de decisión.

En otras palabras, las dificultades y desafíos de la investigación en salud pública están estrechamente relacionados entre sí y se orientan, fundamentalmente, a la superación de los problemas de financiamiento y recursos en general. Pero de base, se encuentra la necesidad de fortalecer una cultura de la investigación en salud pública a nivel país, que favorezca una mayor valoración y compromiso por parte de todos los actores involucrados en el desarrollo de la salud pública en Chile.

Al concluir estas palabras, es oportuno recordar que la Escuela de Salud Pública tuvo desde su creación una fuerte vocación de in- 
fluencia en las políticas públicas de salud, la que dio importantes frutos en la sociedad. Recuperar esta vocación ha sido parte importante de la tarea de estos años: poner a nuestros especialistas y expertos en la opinión pública y al servicio de las grandes tareas del país ha sido uno de los procesos más delicados en que nos hemos involucrado y, como es posible imaginar, no ha sido una tarea fácil.

Pero hoy podemos decir, con mucha confianza, que estamos avanzando en la construcción del círculo virtuoso entre la creación y la sistematización de conocimientos y su aplicación efectiva en el mundo real.

A nombre de la Escuela de Salud Pública de la Facultad de Medicina de la Universidad de Chile, les invito entonces a vivir este Segundo Congreso, a compartir conocimientos, experiencias y miradas, a enriquecer su propio trabajo y a llenarse de energía para continuar aportando al desarrollo de una salud pública que sea capaz de responder oportuna y eficientemente a las necesidades de nuestra gente.

Muchas gracias. 